\title{
The 27-28 October 1986 FIRE Cirrus Case Study: Meteorology and Clouds
}

\author{
David O'C. Starr \\ NASA Goddard Space Flight Center \\ Greenbelt, MD \\ and \\ Donald P. Wylie \\ Space Science and Engineering Center \\ University of Wisconsin \\ Madison, WI \\ Submitted to Monthly Weather Review \\ March, 1989.
}

\begin{abstract}
Detailed descriptions of the rawinsonde-resolved meteorological conditions (3-hourly soundings) associated with a succession of five distinct mesoscale cirrus cloud regimes, that were intensively observed over a 36-hour period, are given. The synoptic scale systems in which these features were embedded are described and a brief overview of the experiment is given. Regional analyses of the static stability structure and vertical motion are presented and interpreted with respect to the characteristics of the corresponding cloud fields as deduced from satellite and lidar observations. The cloud fields exhibited a high degree of persistent mesoscale organization on scales of 20-500 $\mathrm{km}$ reflecting corresponding scales of dynamic and thermodynamic structure/variability as on the synoptic scale. Cloud generation was usually confined to layers less than $1 \mathrm{~km}$ deep (typically $0.5-\mathrm{km}$ in depth) and cellular organization was evident in most cases irrespective of the thermal stratification. Multilayered development was prevalent (2-3 layers) and was associated with vertical structure of the temperature and moisture fields resulting primarily from vertical gradients in horizontal advection. One convective generation layer was usually present. Destabilization resulted primarily from advective processes that also led to the formation of a transient stable layer above and/or below the convective layer. Though resembling elevated frontal surfaces, the stable layers were not extensions of surface features. Cloud processes, primarily ice particle fall-out and evaporation but also including cloud top detrainment, contributed to generating the multilayered structure. Two cases of clouds spawned from an overlying cloud deck were seen where one involved natural seeding of an ice-saturated and conditionally unstable layer in which vigorous convective development was subsequently observed. Subvisual cirrus in the lower stratosphere were found to be associated with prior tropopause features (upwind) where denser cirrus existed. Inferences are drawn with respect to the parameterization of cirrus in large-scale models. In particular, vertical resolution on the order of $0.5 \mathrm{~km}$ will probably be required to adequately resolve the forcing required for implementation of a physically-based parameterization. Greater understanding of the nature and causes of the observed mesoscale structure is also needed.
\end{abstract}




\section{SUMMARY AND CONCLUSIONS}

The cirrus clouds observed on 27-28 October 1986 during the FIRE Cirrus Intensive Field Observation campaign were organized on a wide range of spatial scales. Three synoptic scale systems were identified: the "ridge-crest" cloud, the "ridge-axis" cloud band and the "baroclinic leaf" cloud. Each of these interconnected and long-lived cloud features had a length scale of 1000 $\mathrm{km}$ or more. Relative to the position of a weak low level cyclone, the ridge-crest cloud occurred to the northeast of the cyclone center and surface warm front, the ridge-axis cloud overlay a persistent weak trough in the surface pressure field and the baroclinic leaf cirrus shield was associated with the pre-cold frontal region. However, these extensive cloud systems resulted from upper level processes. Ageostrophic flow (slightly cross-isobaric) within a region extending from just upwind of the large-scale ridge axis (and over the ridge-crest) back toward the following trough axis was responsible for weak ascent and the resultant moisture supply to the large-scale cloud systems. The approximate coincidence of surface features and upper level cloud features reflects their common causes and mutual development rather than indicating a simple cause-effect relationship.

A high degree of persistent mesoscale organization was evident within each of the larger scale cirrus features. At least five distinct mesoscale regimes were intensively observed during the two-day period. Mesoscale uncinus complexes (after Sassen et al., 1989a) in the form of longitudinal bands ( $20 \mathrm{~km}$ in width) along the ambient wind direction but propagating in the transverse direction in correspondence with the movement of the large-scale upper air pattern were observed during the early afternoon of the $27 \mathrm{th}$. They were associated with the entrance region of a strong jet stream core and constituted the leading edge of the ridge-crest cloud that passed mostly to the north of the experiment area. Individual bands were maintained for up to nearly six hours. Later, patches of dissipating cirrus were observed that had formed near the juncture of the three large-scale cirrus systems. During the night, another banded and well-developed mesoscale uncinus complex was observed (Grund and Eloranta, 1989). On the 28th, a large mesoscale cloud system dominated ( $500 \mathrm{~km}$ across). Observations were made of the pre-disturbance cirrus and thin altocumulus and of the denser cirrus and cirriform altocumulus within the disturbance. The large wedge-shaped mesoscale cloud feature was embedded within the synoptic scale baroclinic leaf system and was evident for more than 12 hours. Later, irregular mesoscale patches ( $50 \mathrm{~km}$ across) of significantly enhanced cirrus were observed within the trailing portion of the baroclinic leaf cirrus shield. Other distinct mesoscale cirrus features were also evident as they propagated along the upwind edge of the baroclinic leaf region and had the typical "comma" shape often associated with extensive cirrus shields on the synoptic scale.

The high degree of mesoscale organization in the observed cirrus cloud fields was a manifestation of significant mesoscale structure in the dynamics and thermodynamics of the upper troposphere as shown by our analysis of the available rawinsonde data. Large-scale processes created a favorable environment for the realization of cloud features in association with this structure. The dominance of mesoscale processes, found here and in other recent studies (Sassen et al., 1989a), has significant implications with respect to the parametric representation of cirrus clouds in large-scale atmospheric models. For example, even using three-hourly rawinsonde observations, analyses of the conditions associated with the $500-\mathrm{km}$ disturbance observed on the 28 th were unable to quantitatively diagnose the corresponding forcing (upward vertical motion) although there was strong evidence for ascent based on single station analyses. A judiciously placed special rawinsonde site would probably have allowed us to resolve this forcing. The diagnosed vertical motions for the two regimes observed on the 27 th and the last regime on the 28 th were quite reasonable and likely reflect the smaller amplitude of mesoscale variations of vertical velocity during these events.

Greater understanding of the origin and nature of the mesoscale cloud systems and the responsible dynamic processes is needed. If strong mesoscale organization is an important global 
characteristic of cirrus, this must be taken into account in parametric treatments of cirrus for largescale atmospheric models. We recommend that studies on this topic be initiated.

Another significant conclusion with respect to the structure of the cirrus cloud systems is that cloud generation typically occurred at multiple levels. This tendency for multilayered cloudiness conflicts with the assumption of a single cloud layer that is often invoked in the design of cloud retrieval algorithms for application to satellite observations. Furthermore, the depth of individual generating layers ranged from less than $0.5 \mathrm{~km}$ to about $1 \mathrm{~km}$. If the tendency for multilayered structure and shallow cloud generation layers is widely prevalent, there are important ramifications for modeling cirrus. We suggest that the vertical resolution in mesoscale "cloud process" models (e.g., Starr and Cox, 1985a) should be on the order of $50 \mathrm{~m}$ to adequately capture the true character of the cloud generation and maintenance processes. It is also clear that the vertical resolution of large-scale atmospheric models must also be compatible with the observed scales in order that diagnosis of cirrus generation proceed from physical principles (Starr, 1987a). Otherwise, the parametric treatment must also account for the important effects of unresolved vertical structure of essentially large-scale model parameters in determining cloud response. Based on this case study, a vertical resolution of about $0.5 \mathrm{~km}$ is needed. Substantial improvements in the resolution of jet streams and interactions between the upper and lower troposphere would also very likely result.

Very detailed analyses of the rawinsonde soundings have been presented and compared to qualitative physical descriptions of cloud structure derived from the extensive cloud lidar observations reported in Sassen et al. $(1989 \mathrm{~b})$. In essence, we have attempted to understand why the clouds occurred and what determined their character. This task was greatly complicated by the lack of horizontal resolution of the sonde data. This was compounded by the rather poor vertical resolution of standard rawinsonde data at middle and upper tropospheric levels that made it difficult to unambiguously identify similar features at different locations and times. The enhanced temporal resolution (3-hourly soundings) was crucial and somewhat compensated for the deficiencies in spatial resolution. We also benefited greatly from the availability of high vertical resolution data routinely obtained at a special supplemental rawinsonde site. Despite the inherent uncertainties and the lack of moisture data at high levels, good correspondence between observed thermodynamic and dynamic structure and the cloud observations was found.

The lidar observations reported in Sassen et al. (1989b) revealed that small scale cellular structures $(0.5$ to $2 \mathrm{~km}$ in width) were prevalent in most of the observed cirrus strata. In some cases, we were able to identify a corresponding layer of nearly neutral thermal stratification. When free convection was indicated, it was common to find some cells penetrating significantly into overlying stably stratified layers. A thin cirrostratus layer usually occurred at about the height of the highest cell tops. The underlying layer was often conditionally unstable though generally dry. Evaporation of falling ice crystals (precipitation streamers) may have contributed significantly to its destabilization. However, cellular structures were also found when there was no evidence of convective instability. Maintenance of these structures, likely initiated by shear-induced small scale waves, may have resulted from the effects of latent and radiative heating patterns as shown by Starr and Cox (1985b). Alternatively, local pulsations in microphysical processes (crystal nucleation, growth and fallout) in a sheared environment might be responsible, as shown in Sassen and Dodd (1989). Nonetheless, it is important to note that cellular convective-appearing structure does not always indicate the presence of free convection although it often does. Furthermore, layers of conditional instability are more likely to be associated with the precipitation zone than with the cloud generation layer itself. More-detailed considerations of the nature and origin of observed cellular structure are possible based upon the aircraft observations as in Heymsfield $e t$ al. (1989) and Smith et al. (1989).

Differential (in the vertical) temperature advection appears to have been an important factor in producing the static stability structures that were found to be highly related to cloud generation 
and character. In this respect, the effects of vertical shear of the horizontal wind speed associated with embedded jet flows were paramount on the 27 th while temperature gradients associated with a pool of relatively warm air were important on the 28th. Vertical structure in diabatic heating (and cooling) associated with cloud processes was also significant in this respect. In some instances, the region of cloud formation could be characterized as strongly resembling a frontal overrunning situation, i.e., ascent and destabilization over a sloped elevated layer of substantial static stability. However, the underlying stable layer was transient and not an extension of a surface front. Rather, it appeared to form somewhat in place through a frontogenetic process driven by vertical gradients in horizontal temperature advection.

Evidence was found for cloud-spawned multilayered development resembling the subcloud moistening and destabilization mechanism hypothesized by Starr and Cox (1985b). Late on the 28 th, an initial non-convective cirrus layer appeared to spawn a highly convective cirrus layer below. Natural cloud seeding may have played an important role in the development of the $2-\mathrm{km}$ deep cirriform altocumulus within the mesoscale disturbance on the 28th. Glaciation and growth of large crystals in a stably stratified thin altocumulus layer (pre-disturbance) likely led to natural cloud seeding of an underlying ice-saturated and conditionally unstable layer resulting in cloud formation and vigorous convection extending upward through the initial cloud layer. In each case, generation of the lower cloud layer may be partly attributed to the ice fallout process associated with the presence of relatively large particles in the spawning cloud layer.

Another interesting aspect of this case study is that subvisual cirrus were observed on the 27 th in a stably stratified layer in the lower stratosphere. These "clouds" were a residual (mostly evaporated) of subtropopause cirrus formed upwind in association with a higher tropopause and had advected over the lower tropopause that was forming. The temperature lapse rate in the upwind source region was more tropospheric. The juxtaposition of tropopauses in the vicinity of the subvisual cirrus and the rapidly changing stratification in the intervening layer coincided with the presence of a strong jet core at the lower pause and a secondary wind maximum above

We acknowledge that uncertainties exist in our analyses and interpretations. Nonetheless, the information and insights represent important and unique inputs for model-based efforts to understanding of cirrus. For example, suitably initialized cloud process models may be used to investigate relationships between atmospheric structure and local cloud response for the variety of situations comprising this case. Results of such simulations can be compared to the observed cloud properties to provide a basis for model improvement and validation. Efforts in this area are planned. The available data base will be even greater after additional case studies of the 1986 FIRE Cirrus IFO observations are completed.

Heymsfield, A.J., K.M. Miller and J.D. Spinhime, 1989: The 27-28 October 1986 FIRE Cirrus Case Study: Cloud microstructure. (submitted to Mon. Wea. Rev.)

Grund, C. J., and E.W. Eloranta, 1989: The 27-28 October 1986 FIRE Cirrus Case Study: Cloud optical properties determined by high spectral resolution lidar. (submitted to Mon. Wea. Rev.) Sassen, K., D.O'C. Starr and T. Uttal, 1989a: Mesoscale and microscale structure of cirrus clouds: Three case
studies. J. Atmos. Sci.., 46, 371-396.

Sassen, K., C.J. Grund, J.D. Spinhirne, M. Hardesty and J.M. Alvarez, 1989b: The 27-28 October 1986 FIRE Cirrus Case Study: A five lidar overview of cloud structure and evolution. (submitted to Mon. Wea. Rev.) Sassen, K., and G.C. Dodd, 1989: Haze particle nucleation simulations in cirrus clouds, and applications for
numerical modeling and lidar studies. (submitted to J. Atmos. Sci.)

Smith, W.L., P. Hein and S.K. Cox, 1989: The 27-28 October 1986 FIRE Cirrus Case Study: In situ observations of radiation and dynamic properties of a cirrus cloud layer. (submitted to Mon. Wea. Rev.)

Starr, D.O'C., and S.K. Cox, 1985a: Cirrus clouds, Part I: A cirrus cloud model. J. Atmos. Sci., 42, 2663-2681.

Starr, D.O'C., and S.K. Cox, 1985b: Cirrus clouds, Part II: Numerical experiments on the formation and maintenance of cirrus. J. Atmos. Sci., 42, 2682-2694.

Starr, D.O'C., 1987a: A cirrus cloud experiment: Intensive field observations planned for FIRE. Bull. Amer. Meteoro. Soc., 67, 119-124. 